\title{
The flavour and quark mass dependence of thermodynamic quantities in lattice QCD *
}

\author{
A. Peikert with F. Karsch, E. Laermann \\ Fakultät für Physik, Universität Bielefeld, 33501 Bielefeld, Germany
}

The dependence of thermodynamic properties of QCD on the number of quark flavours is investigated. Lattice results for the equation of state are presented for $2,2+1$ and 3 quark flavours. The simulations have been performed with the improved p4-staggered fermion and a Symanzik improved gluon action on lattices of size $16^{3} \times 4$ and $16^{4}$.

\section{INTRODUCTION}

In this paper thermodynamic observables like the critical temperature $T_{c} / \sqrt{\sigma}$ and the pressure $p / T^{4}$ have been calculated in full QCD with an improved staggered fermion action. The use of improved actions in finite temperature calculations is strongly suggested by results from the pure gauge sector which have shown that improved actions reduce finite cut-off effects in thermodynamic quantities significantly [1,2]. In addition, the calculation of the pressure with the standard staggered action did show strong cut-off effects when comparing results obtained on lattices with temporal extent $N_{\tau}=4$ and 6 [3].

The relevant number of flavours in finite temperature QCD is expected to be two light and one heavier quark. To quantify the effect of varying the number of quark flavours simulations with $N_{f}=2,2+1$ and 3 are performed.

\section{THE SIMULATION}

The fermion fields have been simulated using the tree-level $\mathrm{p} 4$ action [4] including fat-links [5] with a fat-weight $\omega=0.2$ in the one-link derivative. This action is constructed to improve the rotational symmetry of the free quark propagator up to $\mathcal{O}\left(p^{4}\right)$. The high temperature ideal gas limit of the free energy is approached much faster for the $\mathrm{p} 4$ than for the staggered action (Figure 1).

\footnotetext{
*This work was partly supported by the Deutsche Forschungsgemeinschaft under grant Ka 1198/3-1 and the EU TMR network grant ERBFMRX-CT97-0122.
}

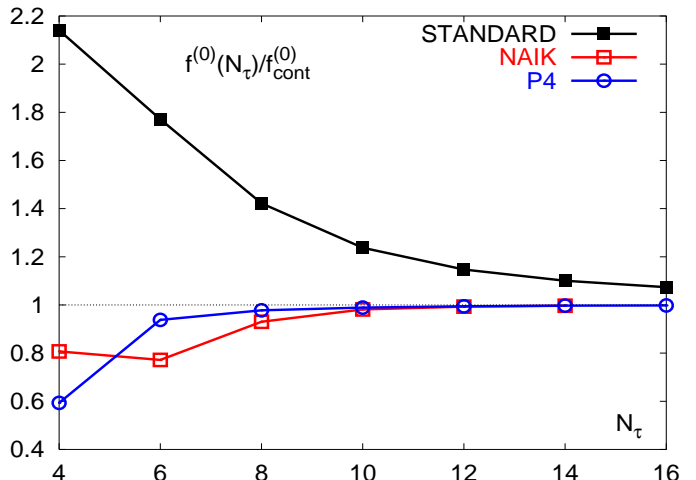

Figure 1. The fermion free energy in the ideal gas limit.

Fat-links lead to an improved flavour symmetry [5] which results in a reduced pion splitting measured by the quantity

$\delta=\frac{m_{\pi_{2}}^{2}-m_{\pi}^{2}}{m_{\rho}^{2}}$.

In Figure $2, \delta$ is shown for the staggered and $\mathrm{p} 4$ fat action measured at different quark masses. Comparing results at the same lattice spacing $a$ one finds a reduction of the pion splitting of about a factor of 2 .

For the gauge fields the tree-level $1 \times 2$ action has been used which in pure gauge simulations lead to an improved finite cut-off behaviour for quantities like latent heat [1] and pressure [2].

The gauge and fermion fields have been updated with the standard Hybrid $\mathrm{R}$ algorithm with a step size $\Delta \tau \lesssim m_{q} a / 2$ and a trajectory length of 0.8. The lattice sizes are $16^{3} \times 4$ and $16^{4}$. 


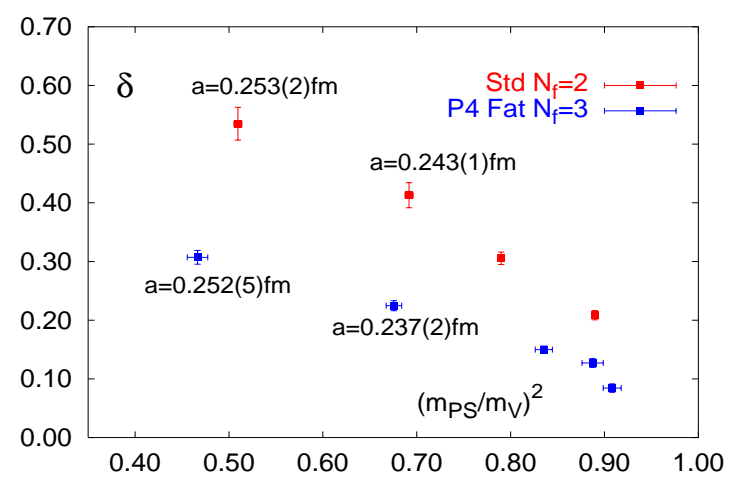

Figure 2. The pion splitting $\delta$ for standard $\left(N_{f}=2\right)$ and p4 fat improved $\left(N_{f}=3\right)$ actions.

\section{THE CRITICAL TEMPERATURE AND THE TEMPERATURE SCALE}

The critical temperature has been calculated for various quark masses for 3 flavour QCD and in addition also for 2 and $2+1$ flavours at a quark mass of $m_{q}=0.10$. The pseudo-critical coupling $\beta_{c}$ was determined by the peak position of the susceptibility of the Polyakov-loop and the chiral condensate, respectively. At the pseudo-critical couplings zero-temperature calculations on $16^{4}$ lattices have been performed measuring the stringtension $\sqrt{\sigma}$ and the meson masses $m_{P S}$ and $m_{V}$. The results for $T_{c} / \sqrt{\sigma}$ are plotted in Figure 3 . The qualitative behaviour of the 2 and 3 flavour values for the standard and $\mathrm{p} 4$ action is quite similar; in both cases $T_{c}$ drops quite fast already

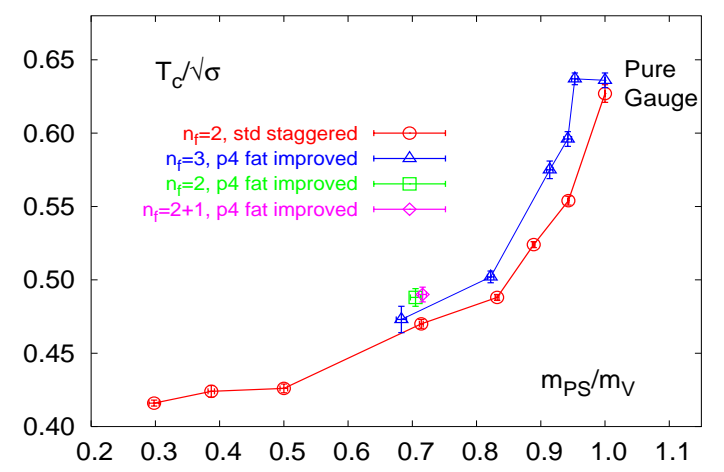

Figure 3. The critical temperature $T_{c} / \sqrt{\sigma}$ for 2 , $2+1$ and 3 flavours for the p4 action. For comparison also 2 flavour results obtained with the standard action [6] are plotted. at large quark masses. For the improved $\mathrm{p} 4$ action $T_{c} / \sqrt{\sigma}$ for different number of flavours does only show a small difference.

To set the temperature scale for the pressure calculations zero temperature simulations have been performed at a variety of $\beta$ values for 2 , $2+1$ and 3 flavours. The resulting string tension data have been fitted to an ansatz proposed by Allton [7] (see Figure 4)

$(a \sqrt{\sigma})(\beta)=R(\beta)\left(1+c_{2} \hat{a}^{2}(\beta)+c_{4} \hat{a}^{4}(\beta)+\ldots\right) / c_{0}$

with $\hat{a}^{2}(\beta)=R(\beta) / R\left(\beta_{0}\right)$, the two-loop beta function $R(\beta)$ and the normalization $\beta_{0}$.

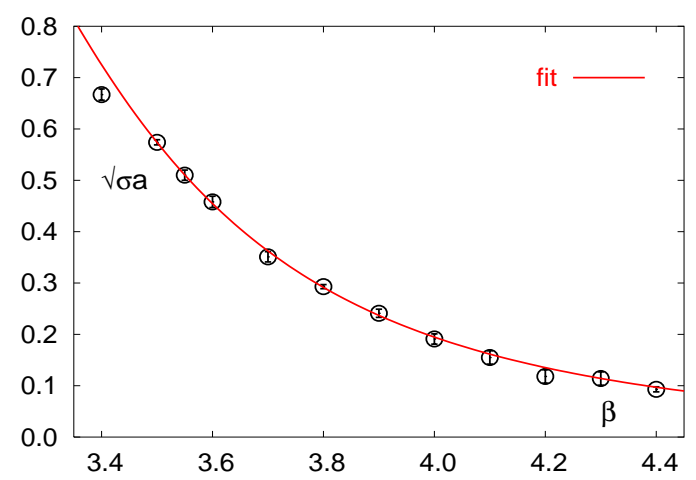

Figure 4. The string tension data and the fit to the data for the $N_{f}=2+1$ case.

\section{THE EQUATION OF STATE}

The pressure $p / T^{4}$ has been calculated for $2,2+1$ and 3 quark flavours. The masses of the light quarks are $m / T=0.4$, the mass of the heavy quark is $m / T=1.0$. From the difference of the gluonic part of the action on finite temperature and zero temperature lattices $\left\langle S^{1 \times 2}\right\rangle_{0}-\left\langle S^{1 \times 2}\right\rangle_{T}$ the pressure can be calculated according to

$\left.\frac{p}{T^{4}}\right|_{\beta_{0}} ^{\beta}=N_{\tau}^{4} \int_{\beta_{0}}^{\beta} d \beta^{\prime}\left(\left\langle S^{1 \times 2}\right\rangle_{0}-\left\langle S^{1 \times 2}\right\rangle_{T}\right)$

In Figure 5 the result for the differences of the gluonic action densities are shown. One observes an increase for the maximum of that quantity corresponding to the increase of the number of flavours. The integration over these curves gives the pressure $p / T^{4}$ plotted in Figure 6. For larger 


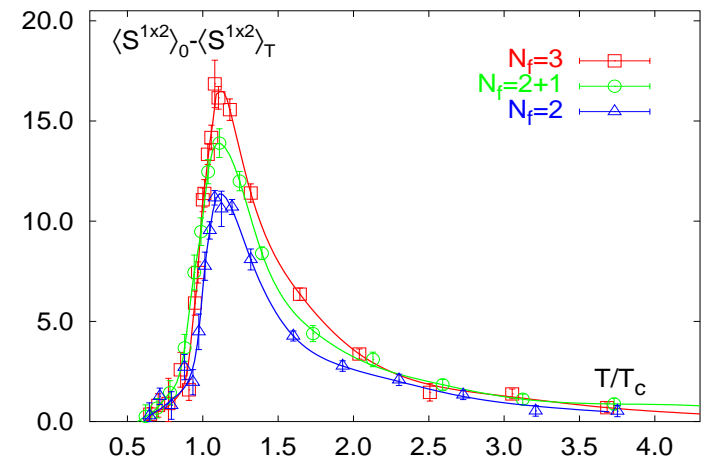

Figure 5. The action differences $\left\langle S^{1 \times 2}\right\rangle_{0}-$ $\left\langle S^{1 \times 2}\right\rangle_{T}$ for $N_{f}=2,2+1$ and 3 for the $\mathrm{p} 4$ fat improved action.

temperatures the $2+1$ flavour pressure is closer to the 3 flavour than to the 2 flavour pressure as expected from the high temperature Stefan Boltzmann limit (see arrows in Figure 6). If one normalizes the pressure to the appropriate ideal gas value one finds the same temperature dependence for 2 and 3 flavours for the p4 action (Figure 7). Figure 7 also shows that the systematic deviations from the Stefan Boltzmann limit obtained with different fermion actions are qualitatively in agreement with what has been calculated as the cut-off effect of the free energy at $N_{\tau}=4$ for the different actions (see Figure 1). The cut-off effects of the 2 flavour pressure for the p4-fat action and the standard staggered action are compared in Figure 8. As the pressure calculated with the $\mathrm{p} 4$ action seems to scale with the relevant number of degrees of freedom we may expect to obtain an estimate for the

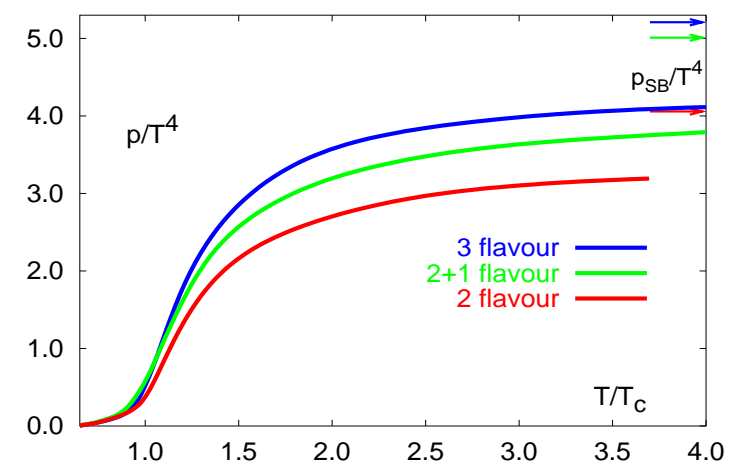

Figure 6. The pressure for $N_{f}=2$, $2+1$ and 3 for the p4 fat improved action.

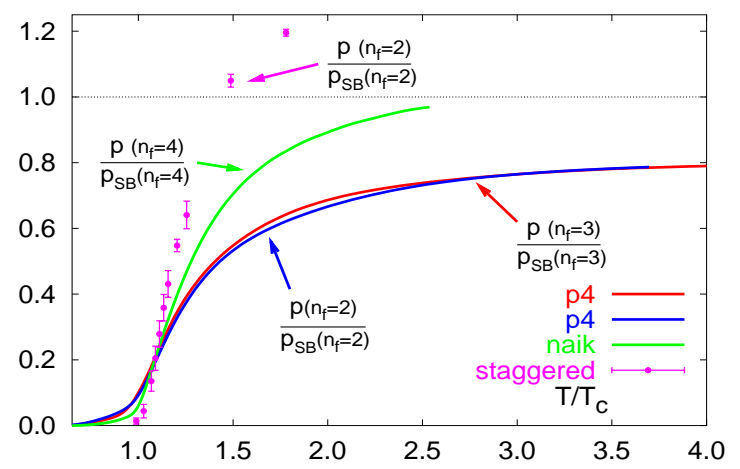

Figure 7. The pressure normalized to the ideal gas value $p / p_{S B}$ for $N_{\tau}=4$.

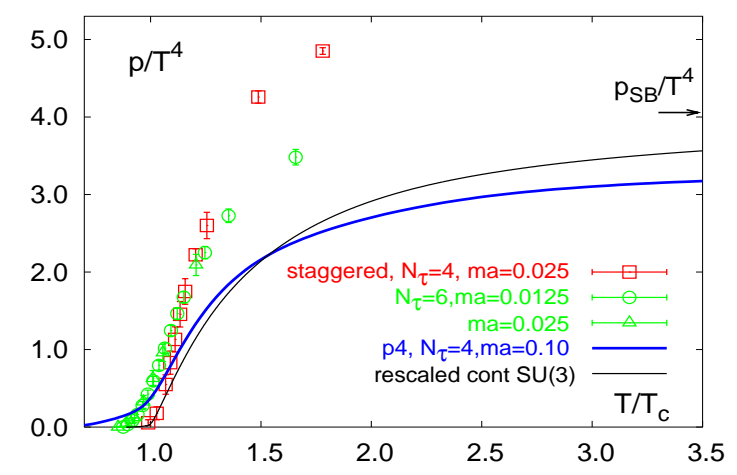

Figure 8. The 2 flavour pressure for the standard action $\left(N_{\tau}=4,6\right)$, the p4 fat action $\left(N_{\tau}=4\right)$ and the rescaled continuum pure gauge result.

remaining cut-off dependence by comparing with the appropriately rescaled continuum extrapolation for the pure gauge pressure. This is shown as the black curve in Figure 8. If this curve approximates the continuum extrapolated pressure of full QCD at high temperatures the p4 action indeed reduces strongly the cut-off effects. A direct verification will require a simulation on a $N_{\tau}=6$ lattice.

\section{REFERENCES}

1. B. Beinlich et al., Phys. Lett. B390 (1997) 268

2. B. Beinlich et al., Eur. Phys. J. C6 (1999) 133

3. C. Bernard et al. (MILC),Phys. Rev D $55(1997) 6861$

4. U.M. Heller et al., hep-lat/9901010

5. T. Blum et al., Phys. Rev. D55 (1997) 1133

6. M. Lütgemeier, Ph.D. thesis, Bielefeld, 1998

7. C. Allton, hep-lat/9610016 